\title{
Supporting information for: \\ Revisiting the non-monotonic dependence of polymer knotting probability on the bending stiffness
}

\author{
Haoqi Zhu ${ }^{1, \#}$, Fujia Tian ${ }^{1, \#}$, Liang Sun ${ }^{1}$, Simin Wang ${ }^{1}$ and Liang Dai ${ }^{1{ }^{1}}{ }^{*}$ \\ ${ }^{1}$ Department of Physics, City University of Hong Kong, Hong Kong, China \\ \#Equal contributions \\ *E-mail: liangdai@cityu.edu.hk
}

Figure $\mathbf{S 1}$ shows the simulation results of a circular polymer with excluded volume interactions and $L=1000 a$. Figure S1a presents the same results of $p_{\text {all }}$ and $p_{k n o t 31}$ as in the main manuscript. In Figure S1b, the probabilities of trefoil knots are converted to the free energies using:

$$
\frac{F_{\text {knot } 31}^{\text {chain }}}{k_{B} T}=-\ln \left(p_{k n o t 31}\right) .
$$

Here, $F_{\text {knot } 31}^{\text {chain }}$ indicates the relative free energy cost of forming a trefoil on the entire polymer. We shift the first point of the curve of $F_{\text {knot } 31}^{\text {chain }}$ to zero so that $F_{\text {knot } 31}^{\text {chain }}$ are relative to the value at $L_{p}=0$. Figure S1c plots the simulation results of the extra bending energy induced by knotting, $\Delta E_{\text {bend }}$, which is defined as the difference in the average bending energy between trefoil-knotted polymers and unknotted polymers:

$$
\Delta E_{\text {bend }} \equiv\left\langle E_{\text {bend }}^{\text {knot } 31}\right\rangle-\left\langle E_{\text {bend }}^{\text {unknot }}\right\rangle
$$

For $L_{p} \lesssim 8 a, \Delta E_{\text {bend }}$ is negative, which indicates that knotting reduces the polymer bending energy. The negative values of $\Delta E_{\text {bend }}$ have been observed and discussed in several previous studies [1-3].

Figure S2 shows the simulation results of a circular polymer with $L=1000 a$ in the absence of excluded volume interaction. Figure $\mathbf{S 2 a}$ presents the same results of $p_{\text {all }}$ and $p_{\text {knot } 31}$ as in the main manuscript. Figure $\mathbf{S 2} \mathbf{b}$ shows the relative free energy cost of forming a trefoil knot on the entire polymer. Because $L_{k n o t}^{*} / L_{p}$ remains unchanged upon varying $L_{p}$, the free energy cost of forming a trefoil knot on a fragment, $F_{\text {frag }}$, is insensitive to $L_{p}$. Accordingly, the changed in $F_{\text {knot } 31}^{\text {chain }}$ is mainly caused by the knot-size effect, i.e. $F_{\text {knot } 31}^{\text {chain }} \sim-\ln \left(N_{\text {frag }}\right) \sim \ln \left(L_{p}\right)$. The red line in Figure S2b captures the simulation results for large $L_{p}$. Figure S2c plots $\Delta E_{\text {bend }}$ as a function of $L_{p}$. When $L_{p}$ decreases from 20 a to $8 \mathrm{a}$, the change in $\Delta E_{\text {bend }}$ is about $-0.34 k_{B} T$, while the change in $F_{k n o t 31}^{\text {chain }}$ is about $-1.04 k_{B} T$. These results suggest that the change in the bending energy should not be the leading reason for the change in the knotting probability upon varying $L_{p}$.

Figure S3 shows the simulation results of the knot spectrum of a circular polymer with $\mathrm{L}=1000 \mathrm{a}$ in the presence of excluded volume interaction. 


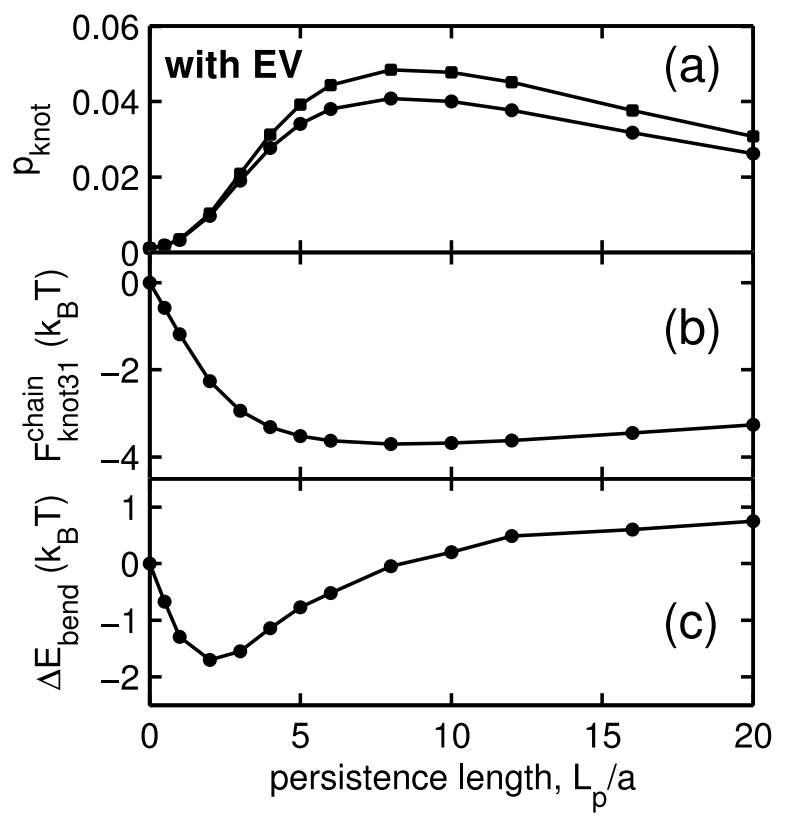

Figure S1. Simulation results of a circular polymer with excluded volume interactions and $L=1000 a$. (a) The probabilities of all knots (squares) and trefoil knots (circles) as a function of the persistence length. (b) The free energy cost of forming a trefoil knot on the entire polymer calculated from the simulation results of $p_{\text {knot } 31}$. Note that we shift the first point of the curve to zero. (c) Simulation results of the differences in the average bending energy between knotted and unknotted polymer conformations. 


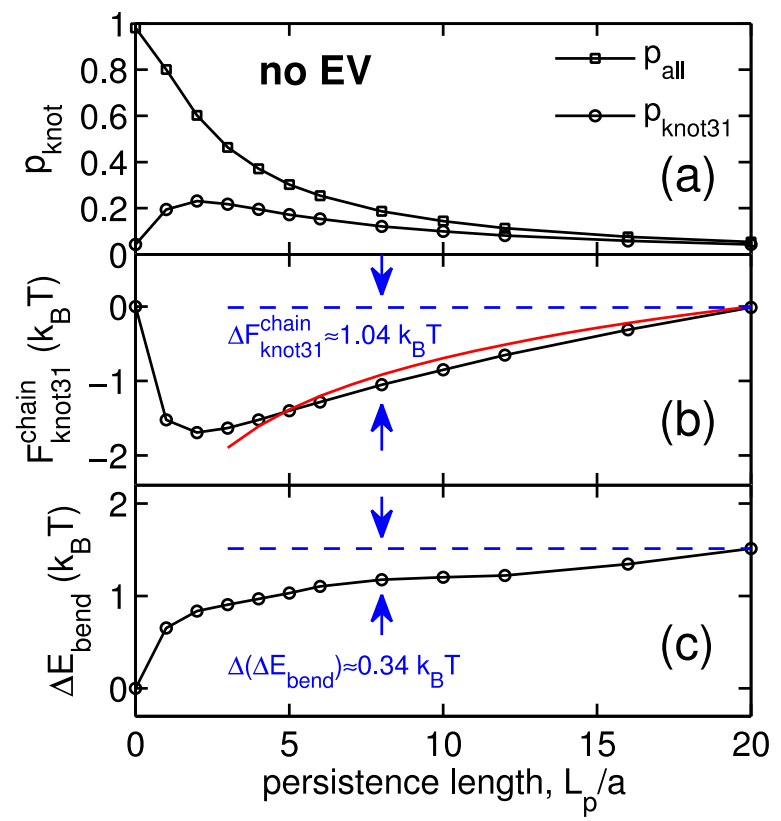

Figure S2. Simulation results of a circular polymer with $L=1000 \mathrm{a}$ in the absence of excluded volume interaction. (a) The probabilities of all knots (squares) and trefoil knots (circles) as a function of the persistence length. (b) The free energy cost of forming a trefoil knot on the entire polymer calculated from the simulation results of $p_{\text {knot } 31}$. Note that we shift the first point of the curve to zero. The difference in $F_{\text {knot }}^{\text {chain }}$ at $L_{p} / a=8$ and $L_{p} / a=20$ is about $1.04 k_{B} T$. The red line corresponds to $y=-\ln (x / 20)$. (c) The difference in the average bending energy between knotted and unknotted polymer conformations. The difference in $\Delta E_{\text {bend }}$ at $L_{p} / a=8$ and $L_{p} / a=20$ is about $0.34 k_{B} T$. 

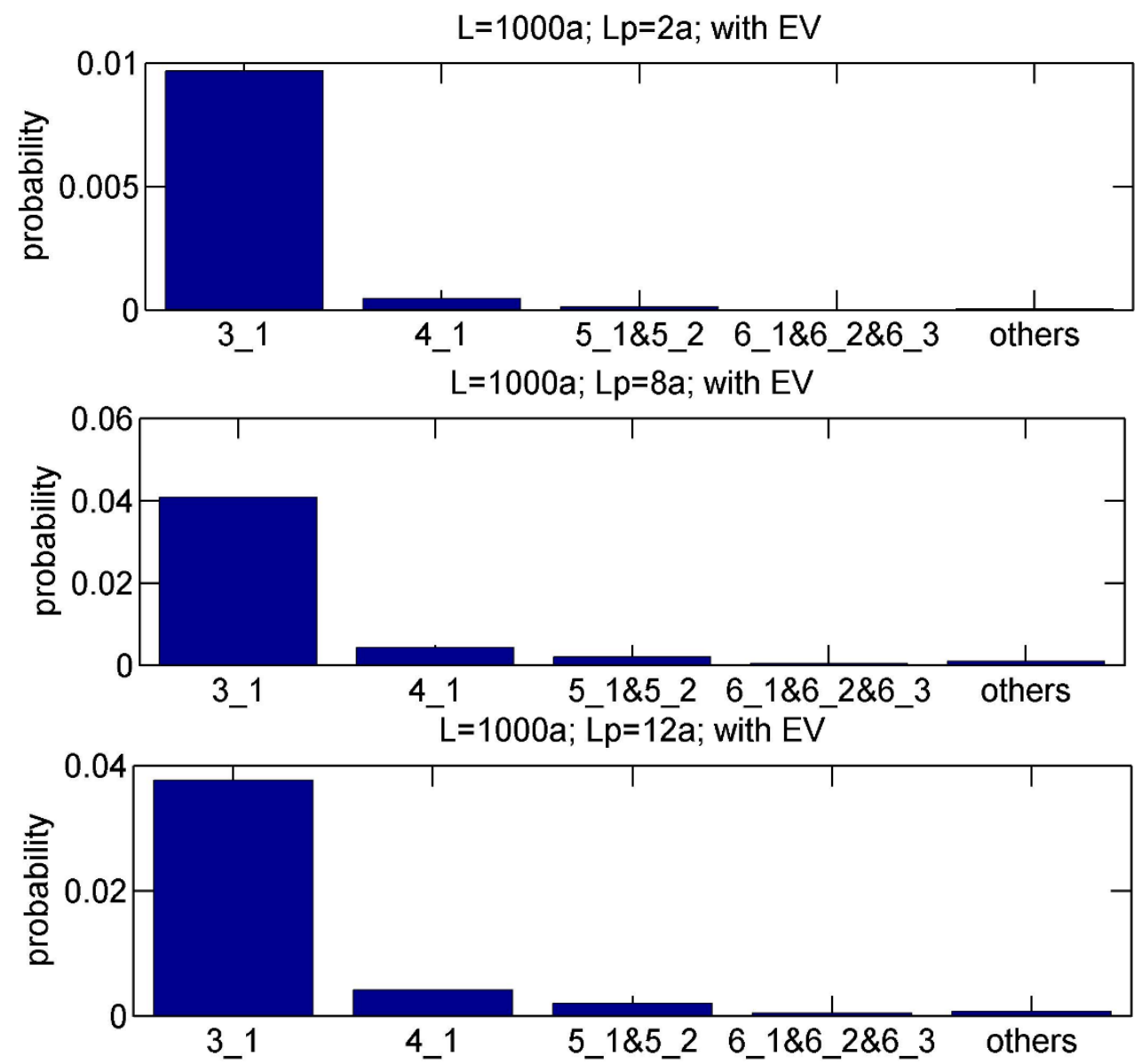

Figure S3. Simulation results of the knot spectrum of a circular polymer with $L=$ $1000 a$ in the presence of excluded volume interaction. The three plots correspond to three values of persistence length: $L_{p}=2 a$ (top), $L_{p}=8 a$ (middle), and $L_{p}=12 a$ (bottom).

1. Poier, P.; Likos, C. N.; Matthews, R., Influence of Rigidity and Knot Complexity on the Knotting of Confined Polymers. Macromolecules 2014, 47, 3394.

2. Coronel, L.; Orlandini, E.; Micheletti, C., Non-monotonic knotting probability and knot length of semiflexible rings: the competing roles of entropy and bending energy. Soft Matter 2017, 13, 4260-4267.

3. Lu, L.; Zhu, H.; Lu, Y.; An, L.; Dai, L., Application of the Tube Model to Explain the Unexpected Decrease in Polymer Bending Energy Induced by Knot Formation.

Macromolecules 2020, 53, 9443-9448. 